\title{
Lower Limb Motion Recognition Method Based on Improved Wavelet Packet Transform and Unscented Kalman Neural Network
}

\author{
Xin Shi $\mathbb{D}^{1,2}$ Pengjie Qin ${ }^{1 D},,^{1,2}$ Jiaqing Zhu $\mathbb{D}^{1,2}$ Shuyuan Xu ${ }^{1 D},{ }^{1,2}$ and Weiren Shi $^{3}$ \\ ${ }^{1}$ Institute of Automation, Chongqing University, Chongqing 400044, China \\ ${ }^{2}$ Key Laboratory of Complex System Safety and Control, Ministry of Education, Chongqing 400044, China \\ ${ }^{3}$ Shenzhen Institute of Artificial Intelligence and Robotics for Society, Shenzhen, China
}

Correspondence should be addressed to Xin Shi; shixin@cqu.edu.cn

Received 1 December 2019; Revised 22 February 2020; Accepted 2 March 2020; Published 27 April 2020

Academic Editor: Rafal Zdunek

Copyright (C) 2020 Xin Shi et al. This is an open access article distributed under the Creative Commons Attribution License, which permits unrestricted use, distribution, and reproduction in any medium, provided the original work is properly cited.

Exoskeleton robot is a typical application to assist the motion of lower limbs. To make the lower extremity exoskeleton more flexible, it is necessary to identify various motion intentions of the lower limbs of the human body. Although more sEMG sensors can be used to identify more lower limb motion intention, with the increase in the number of sensors, more and more data need to be processed. In the process of human motion, the collected sEMG signal is easy to be interfered with noise. To improve the practicality of the lower extremity exoskeleton robot, this paper proposed a wavelet packet transform- (WPT-) based sliding window difference average filtering feature extract algorithm and the unscented Kalman neural network (UKFNN) recognition algorithm. We established an sEMG energy feature model, using a sliding window difference average filtering method to suppress noise interference and extracted stable feature values and using UKF filtering to optimize the neural network weights to improve the adaptability and accuracy of the recognition model. In this paper, we collected the sEMG signals of three muscles to identify six lower limb motion intentions. The average accuracy of $94.83 \%$ is proposed in this paper. Experiments show that the algorithm improves the accuracy and anti-interference of motion intention recognition of lower limb sEMG signals. The algorithm is superior to the backpropagation neural network (BPNN) recognition algorithm in the lower limb motion intention recognition and proves the effectiveness, novelty, and reliability of the method in this paper.

\section{Introduction}

Surface electromyography (sEMG) signals are bioelectrical signals recorded from the surface of the muscle through the electrode. It has the advantages of mature acquisition technology, high temporal resolution, and noninvasive recording [1-3]. In addition to some significant achievements in medical rehabilitation training, relevant research applications have gradually developed into a wider range of lower limb motion assist areas [4]. The most common lower extremity exoskeleton robots currently include HAL exoskeleton robot [5], MIT exoskeleton system [6], BLEEX lower limb exoskeleton [7], and NTULEE exoskeleton system [8]. The robot can walk horizontally, but it has few motion intention and low flexibility. Therefore, identifying more lower limb motion intention is beneficial to improve flexibility and practicability. It can also enhance the user's athletic ability and improve people's quality of life.

Most of the current research is based on sEMG signals to identify multiple motion intentions in the lower extremities. Coelho and Lima extracted eight channels of sEMG signals to identify seven motion intentions for the lower extremities [9]. Toledo-Pérez et al. collected sEMG signals from four muscles of the lower extremities and identified ankle dorsiflexion and flexion [10]. Al-Quraishi et al. extracted the sEMG signal from the four muscles of the lower extremities and identified four motion intentions of the lower extremity ankle joint [7]. Tang et al. collected three channels of sEMG signals and identified three intentions of standing, flexing, and stretching [11]. Ma et al. collected sEMG signals of the 
rectus femoris $(\mathrm{RF})$, semimembrane $(\mathrm{SM})$, and the sphincter (SR) and identified two intentions of walking and obstacle crossing [12]. Li et al. recorded the sEMG signals of seven muscles of the lower extremities and recognized two motion intentions of the hip and knee joints of the lower extremities [13]. Ai et al. combined acceleration and sEMG signals to identify five motion intentions of the lower extremities [14]. To identify the lower extremity knee, ankle, and hip joint motions, Tapia et al. extracted sEMG signals from sixteen muscles of the lower extremities [15]. Zhang et al. extracted single channel sEMG signals for four motion intention recognition of lower limb knee joints [16]. These studies did not simultaneously consider accuracy, stability, and realtime. Therefore, we proposed a method of using three electromyographic sensors to recognize six kinds of lower limb motion intention.

The process of lower limb motion intention recognition mainly includes feature extraction and motion recognition. For example, some researchers used RMS [17, 18], mean absolute value (MAV) [19], autoregressive (AR) [20], variance, and Willison amplitude method to extract surface muscle electrical signal features [21, 22]. Some researchers used peak frequency and median frequency analysis [23, 24] to convert sEMG signals to the frequency domain and extracted sEMG features.

However, the sEMG signal is easily disturbed by static electricity, muscle fatigue, and friction factors. Traditional feature analysis methods cannot accurately describe the time-varying process and spectrum distribution of the sEMG signal, which leads to low reliability of feature extraction. WPT is a common time-frequency analysis method, which can reflect the energy distribution of the sEMG signal in each frequency band [25]. Gokgoz et al. used the WPT method to extract time-frequency information of myoelectric signals and diagnosed the lower limb neuromuscular diseases. Chen et al. proposed a wavelet packet of local energy (ELF) method to accurately describe the complexity in the local frequency band of myoelectric signals [26]. Ji et al. used discrete wavelet transform to construct a time-invariant multiscale matrix based on wavelet transform coefficients to identify eight motion intentions of lower limbs [27]. However, due to noise interference, the feature extracted by the wavelet packet cannot accurately describe the motion intention of lower limbs. Therefore, we proposed a sliding window differential average filtering method, analyzing the feature extracted by wavelet packet transform, suppressing noise interference, and extracting effective feature vector.

In the part of the lower extremity motion intent recognition, SEMG signal has complex nonlinear, strong coupling, and dynamic time-varying features [28]. In the aspect of motion intention recognition, researchers mainly studied the Bayesian network, neural network, multilayer perceptron, fuzzy approximation, support vector machine, and neural fuzzy system identification method [29, 30]. Neural network has strong nonlinear approximation ability and the ability to deal with unknown internal mechanism problems [31-33]. However, the neural network model needs iterative learning in the training process [34], the convergence speed is slow, and the real-time performance needs to be improved $[35,36]$. The sEMG signal is susceptible to external environmental influences during the acquisition process, such as sweating, muscle fatigue, electrode offset, and power frequency noise [37, 38]. The neural network model lacks stability when the environment changes. Therefore, we proposed an unscented Kalman neural network (UKFNN) method for lower extremity motion intention recognition. We used the UKF method $[39,40]$ to train the weight of the neural network to enhance the adaptive ability of the recognition model [41]. Our method improves the reliability, accuracy, and speed of the model.

We extracted sEMG signals from three channels and used the WPT method to extract features. We proposed a sliding window difference averaging filtering method to analyze the time-frequency domain feature, suppress noise interference, and extracted effective feature information. We constructed a UKFNN recognition model to recognize six lower limb motion intentions. Also, we compared the proposed recognition algorithm with the error backpropagation neural network (BPNN) recognition method [42] and evaluated the recognition accuracy of UKFNN.

This paper is structured as follows. The second part described the acquisition of myoelectric signals. The third part introduced the extraction method of sEMG signal features and explained the basic principle of the wavelet packet transform. Also, we proposed a feature analysis method for sliding window difference averaging filtering. The fourth part introduced the design of the UKFNN model. The fifth part gave the experimental results and compared them with the traditional BPNN method. The sixth part gives conclusions and future work.

\section{2. sEMG Acquisition}

Surface electromyography is recorded from the surface of human skeletal muscle through surface electromyography electrodes and contains many feature information related to limb motion. By analyzing these features, we can distinguish the different motion intentions of the limbs. Our goal is to use three sensors to identify six lower extremity motion intentions. We chose six common motion intentions of lower limbs, such as horizontal walking (HW), crossing obstacles (CO), standing up (SU), downstairs (DS), goupstairs (GU), and stop-rest (SR). We analyzed the kinematics and biological features of lower limb muscles $[43,44]$. The medial gastrocnemius (mg) is helpful for walking and running. The femoral muscle (VL) and the semitendinosus (ST) have the function of flexing the knee joint and stretching the hip joint [45]. Therefore, we selected three muscles as the source of sEMG signal acquisition initially, as shown in Figure 1.

We used three sEMG sensors to identify six lower limb motion intentions. The six motion intentions were $\mathrm{HW}, \mathrm{CO}$, SU, DS, GU, and SR, as shown in Figure 2.

We used the RMS method to calculate the changing trend of the sEMG signal of three muscles in six motion intentions, as shown in Figure 3. 

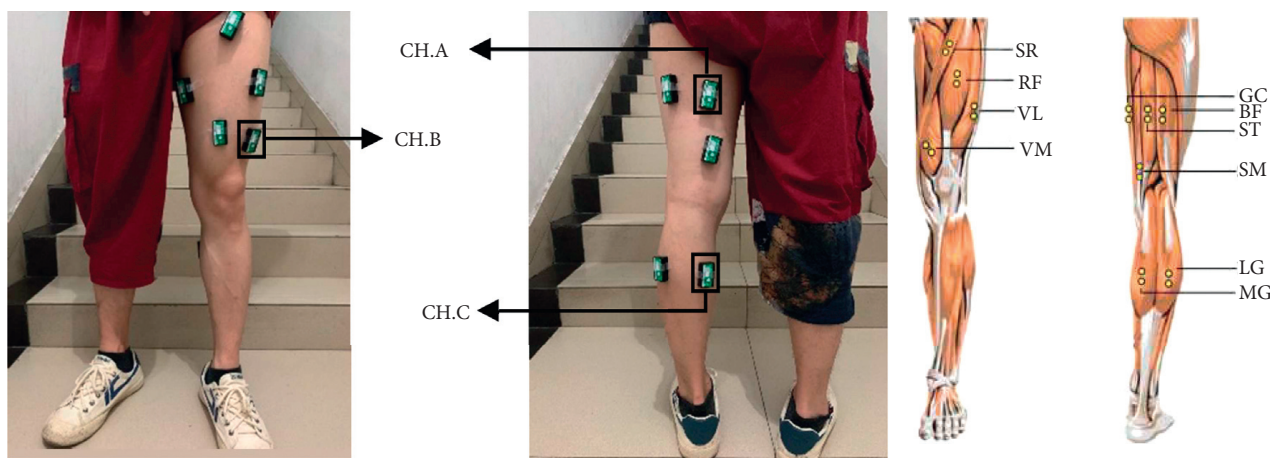

Figure 1: sEMG signal sensor location. Channel A is located in the thigh semitendinosus, channel B is located in the lateral thigh muscle, and channel $\mathrm{C}$ is located in the calf gastrocnemius.

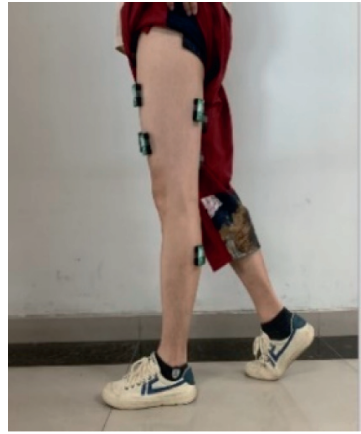

(a)

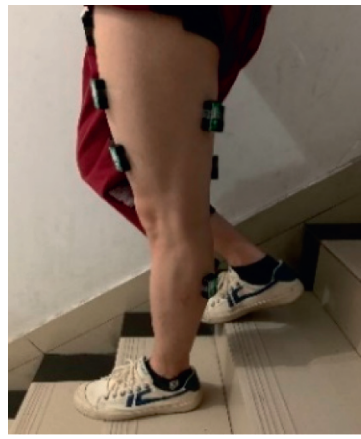

(d)

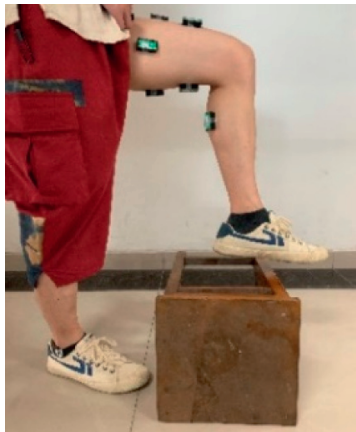

(b)

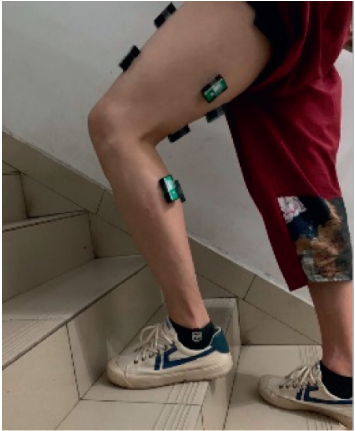

(e)

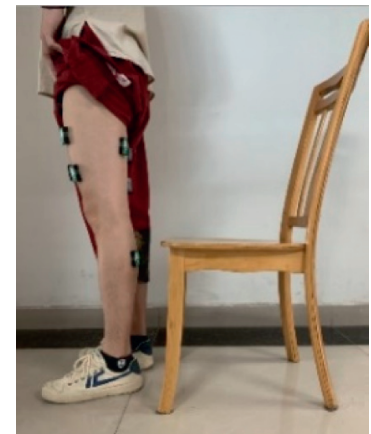

(c)

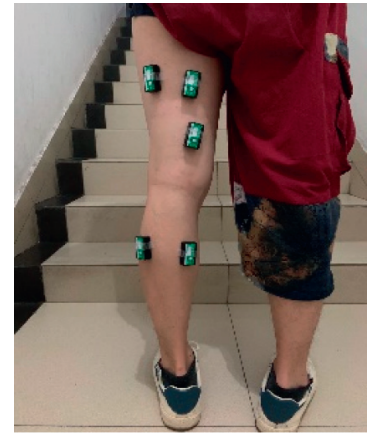

(f)

FIgURE 2: Sis intentions of lower limb motion: (a) HW; (b) CO; (c) SU; (d) DS; (e) GU; (f) SR.

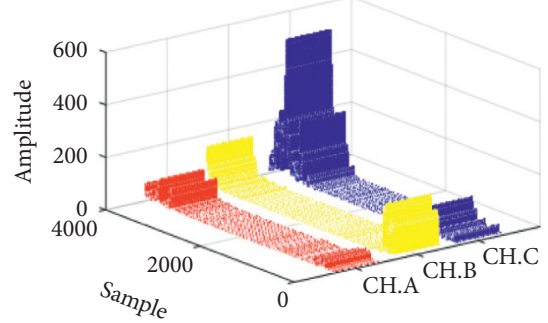

(a)

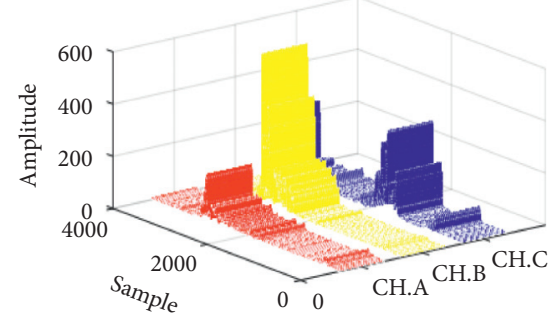

(b)

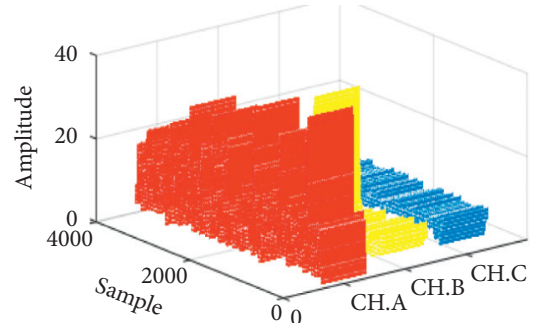

(c)

Figure 3: Continued. 


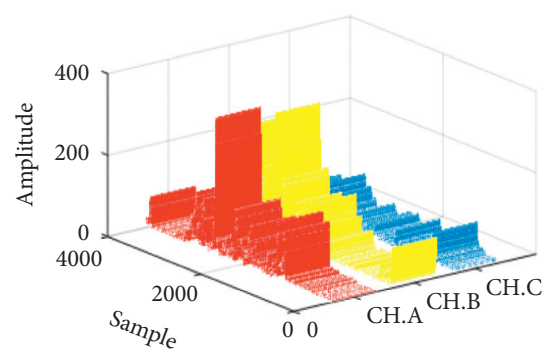

(d)

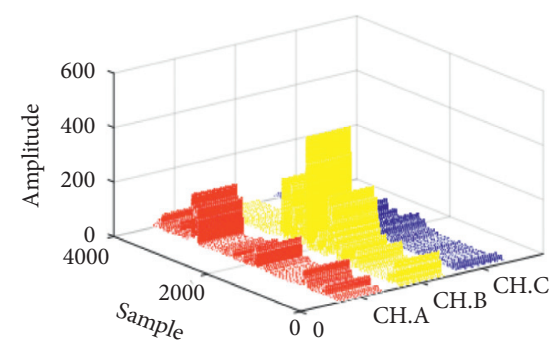

(e)

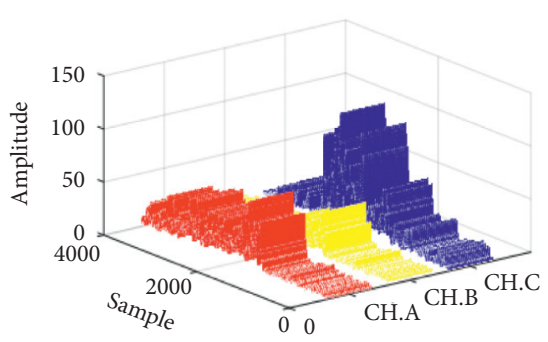

(f)

FIgURE 3: RMS features of three muscles in six motion intentions: (a) CO; (b) HW; (c) SR; (d) GU; (e) DS; (f) SU.

It can be seen that the selected three muscles have obvious signal changes during the motion of the lower limbs. The changing trend of sEMG signals in three channels of a motion mode is the same. However, the trend of sEMG signal changes in six motion intentions is different. This is helpful to distinguish different motion intentions and further verifies the correctness of the selection of sEMG signal acquisition location.

We used the sEMG sensor developed by Biometrics, UK. The sampling frequency is $2000 \mathrm{~Hz}$, and the input impedance of the amplifier is more than 10,000,000 $\mathrm{m}$ ohms. Highquality signals can be obtained without adding conductive adhesive to the skin. Simply attach the electrode strip to the three muscles using medical double-sided tape and record the surface myoelectric signal. The experimental computing platform processor is Intel(R) Core(TM) i7-9750H CPU@ $2.60 \mathrm{GHz}$, the memory is $16 \mathrm{G}$, and the data analysis software is Matlab, 2015b.

We selected five healthy subjects to participate in the experiment, five men aged $23,23,25,26$, and 24 years, with a body fat rate of $17 \pm 3 \%$ and a height of $170 \pm 5 \mathrm{~cm}$. Healthy subjects circulate in the form of "relaxation-motion-relaxation" for each motion, completing six motion intentions. Each motion is limited to two seconds. We collected sEMG signals from six intentions of lower extremity motions per subject.

\section{3. sEMG Feature Extraction Method}

The acquired sEMG signals have nonperiodic, nonstationary, and nonlinear chaotic features. We need to extract stable eigenvectors from myoelectric signals. Therefore, this paper used the wavelet packet transform analysis method, to describe the complex sEMG signal by an energy method. We proposed a sliding window difference average filtering method which can suppress the noise interference and extract the effective sEMG eigenvector.

(1) The research shows [26] that the sEMG signal should be decomposed into three layers. The decomposition structure is shown in Figure 4.

(2) We used wavelet decomposition coefficients to reconstruct the signal and analyzed all nodes in the third layer. The raw signal can be restored to

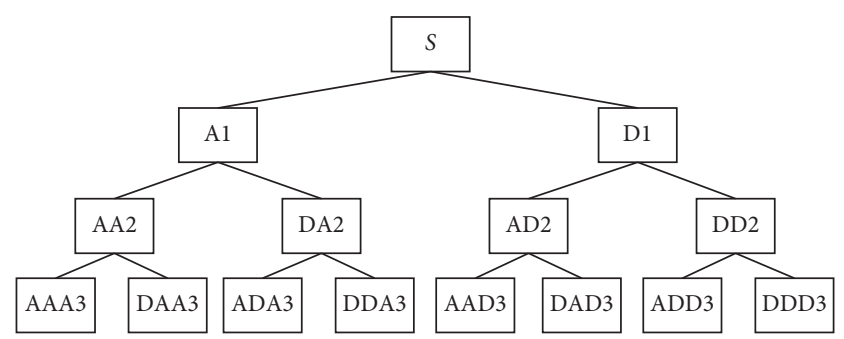

FIgURE 4: Three-layer wavelet packet decomposition tree, where $S$ is the raw signal, $A$ represents a low-frequency signal, and $D$ represents a high-frequency signal. The number indicates the number of layers of the wavelet decomposition. We extracted the signal features of the third layer, a total of eight frequency components.

$$
\begin{aligned}
S= & \mathrm{AAA}_{3}+\mathrm{DAA}_{3}+\mathrm{ADA}_{3}+\mathrm{DDA}_{3}+\mathrm{AAD}_{3}+\mathrm{DAD}_{3} \\
& +\mathrm{ADD}_{3}+\mathrm{DDD}_{3} .
\end{aligned}
$$

(3) Define wavelet packet energy as $E_{j}^{i}$, the coefficients of wavelet packet decomposition as $f_{j}^{i}(t)$, and the constructed feature vector as $\left[E_{j}, E_{j-1}, \ldots, E_{1}\right]$ :

$$
E_{j}^{i}=\int_{-\infty}^{+\infty} f_{j}^{i}(t)^{2} \mathrm{~d} t
$$

where $i=3$ represents the scale and $j=8$ represents the node.

(4) Normalizing the energy of eight nodes:

$$
\begin{gathered}
E_{\mathrm{tot}}=\sum_{i=1}^{2^{j}} E_{j}^{i}, \\
P_{i}=\frac{E_{j}^{i}}{E_{\mathrm{tot}}} .
\end{gathered}
$$

3.1. Wavelet Packet Transform. The energy value of WPT can represent the changing trend of complex sEMG signals. The steps of the wavelet packet energy feature extraction algorithm are as follows. 
3.2. Mother Wave Function Selection. Selecting different wavelet packet basis functions will get different eigenvalues, which will directly affect the recognition accuracy of lower limb motion intention. It is very important to select the appropriate basis function for extracting features. The energy feature values obtained by the wavelet packet transform should contain enough information on the raw signal to ensure the recognition accuracy. The larger the wavelet coefficient is, the similar the base function waveform is to the raw signal. Hence, selecting a basis function with high similarity to the raw signal contributes to the extraction of features. As shown in Figure 5, the raw signal is nonstationary and nonperiodic, with strong tightness.

We observed the wavelet packet basis function waveform in Figure 6. We can see the waveforms of sym3, sym5, cmor1-1.5, and the waveform of the myoelectric signal are different, and local performance is poor. The Harr wavelet is not continuous in the time domain and is not suitable for sEMG signal feature extraction. The attenuation rate of $\mathrm{db} 8$, $\mathrm{db} 10$, coif3, and $\mathrm{fk} 22$ is slow. It is easy to ignore the small change information in the raw sEMG signal [46]. The waveform of the dmey wavelet is similar to the raw sEMG signal, which has strong compactness and fast attenuation performance. Therefore, using the dmey wavelet to extract feature values is beneficial to improve the recognition accuracy of motion intention.

To extract the raw signal features of sEMG accurately, the wavelet packet basis function is with the maximum energy value in all motions. As shown in Figure 7, the average energy value of the dmey wavelet packet basis function is the highest, which further verifies the correctness of the wavelet packet basis function selection.

3.3. sEMG Feature Selection. At present, most studies choose the maximum absolute value of wavelet coefficients as the eigenvector of sEMG signals $[25,26]$. However, in different motion intentions, the amplitude of the sEMG signal on the same muscle is different. Choosing wavelet coefficients as eigenvalues cannot accurately reflect the motion intention. We used the wavelet packet to decompose the sEMG signal, normalizing the complex sEMG signal to different frequency bands, and analyzed the energy values of the projection sequences in each band. We used a three-layer wavelet packet to decompose the sEMG signal of the gastrocnemius, as shown in Figure 8. Also, we calculated the sEMG signal energy eigenvalues of three muscles in six motions, as shown in Table 1.

Observing the wavelet packet energy values of each node in Table 1, we analyzed the energy distribution of six motion intentions of the same muscle and the energy distribution of three muscles in the same motion mode. It can be concluded that the energy values of $\mathrm{AAA}_{0}, \mathrm{DAA}_{1}, \mathrm{ADA}_{2}$, and $\mathrm{DDA}_{3}$ are significantly different from those of other frequency bands, as shown in Figure 9. Therefore, we chose four subband energy values as eigenvectors. The eigenvector for each motion is twelve dimensions. We have extracted the 12dimensional eigenvectors and built a feature model to analyze the eigenvectors, as shown in Figure 10.
Considering that sEMG signals are susceptible to noise interference during motion, such as sweating, friction, and electrode deflection, as shown in Figure 11, the sEMG signals of different muscles fluctuate aperiodically [37, 38].

We have built a feature model containing the interfering signals, as shown in Figure 12.

It can be seen from Figure 12 that the feature model of different motion intentions including interference signals is a periodic signal. But they have different peaks and troughs in the same period. We calculated the eigenvector to suppress the interference of noise. As shown in Figure 13, we set the width of the sliding window as $M=2$ and the sliding step size as $D=4$. Get three window feature values. We calculated the difference between the maximum value and the minimum value of the energy feature in each window and calculated the average values of the three differences.

We calculated and analyzed the difference average for each motion, as shown in Table 2 .

Through the proposed sliding window difference average filtering algorithm, we calculated the eigenvalue in Figure 12 and analyzed the results of different noise interferences, as shown in Table 3. Therefore, we can set a threshold. If the difference average of eigenvalues is greater than 90, it can be judged that the sEMG is interfered with noise. We extracted feature vector by judging the threshold and removed the disturbing feature vector.

Our proposed sliding window difference average filtering method suppresses the interference of noise and extracts a stable feature vector. The 12-dimensional feature vector filtered by the sliding window difference average can be the input of the UKFNN to improve the reliability of the recognition model.

\section{Recognition Using UKFNN}

In the lower limb motion intent recognition algorithm, because the neural network has a strong nonlinear fitting ability, it is widely used in the modeling and optimization of the motion intention recognition process [33]. However, the lower limb sEMG signal has complex nonlinearity, strong coupling, and dynamic time-varying features during motion. The signal is susceptible to noise such as sweating, electrode offset, and power frequency interference $[37,38]$. This leads to a lack of stability in the neural network model. Using UKF filtering for neural network learning can enhance the adaptive ability of the model and raise the accuracy of the model. Especially when the number of samples is increasing, it can speed up the convergence of the model [40]. Therefore, we used the UKFNN algorithm [41, 47-49] to recognize the motion intention of lower limbs. We used the UKF algorithm to optimize the weight of the neural network, which can adapt to noise interference.

As shown in Figure 14, the UKFNN structure is a threelayer neural network.

The input of UKFNN is an $\mathrm{N}$-dimensional eigenvector, and the input in Figure 14 is defined as $X$ :

$$
X=\left[x_{1}, x_{2}, x_{3}, \ldots, x_{n}\right] \text {. }
$$

The output of the UKFNN network in Figure 14 is defined as $Y$ : 


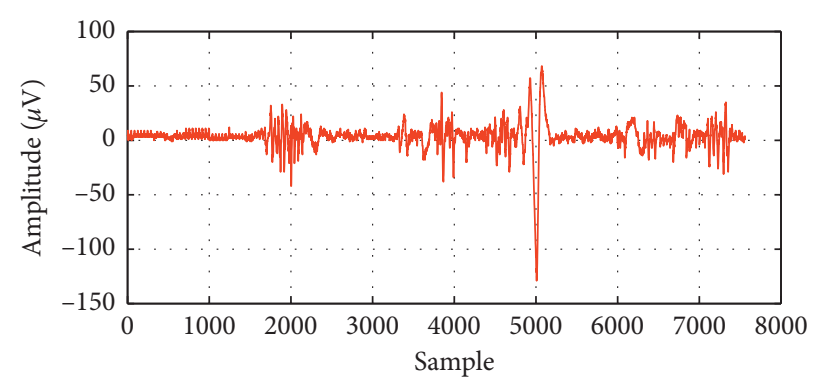

(a)

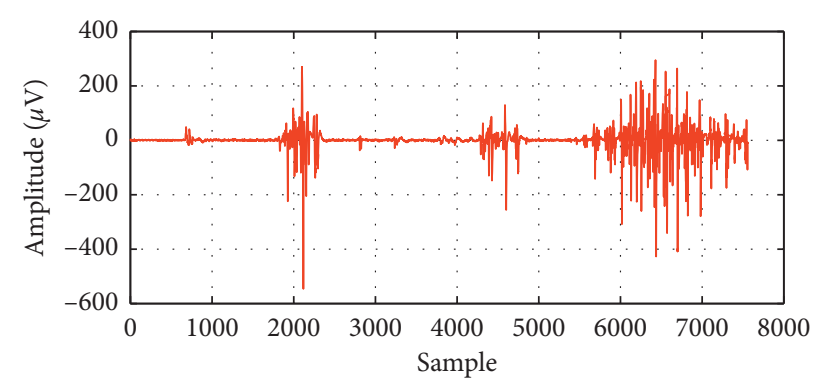

(b)

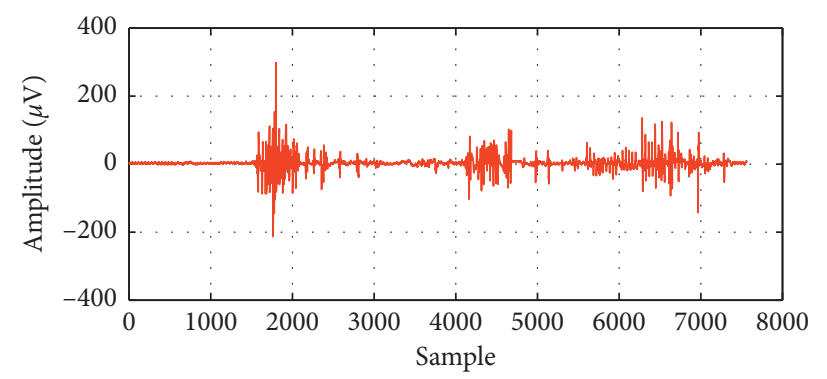

(c)

FIGURE 5: sEMG signals of three muscles in the walking mode.

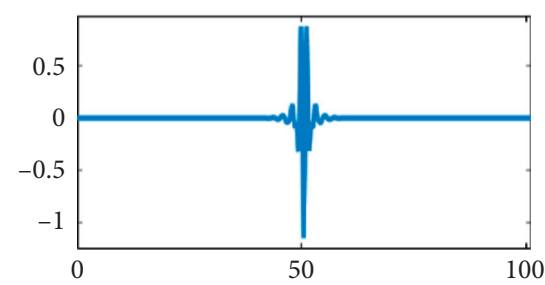

(a)

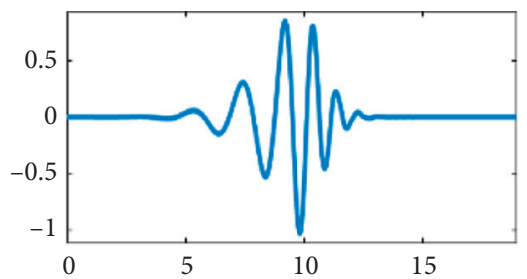

(d)

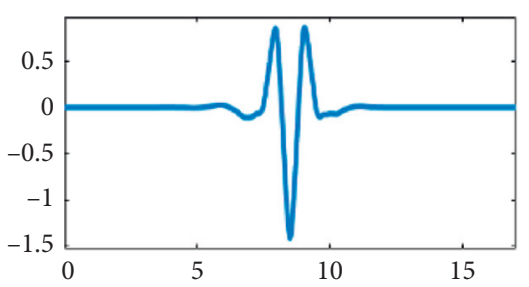

(g)

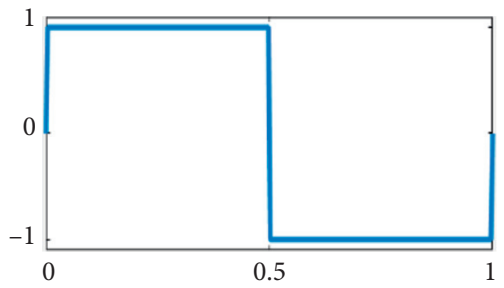

(b)

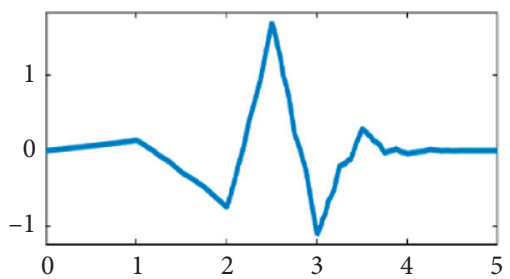

(e)

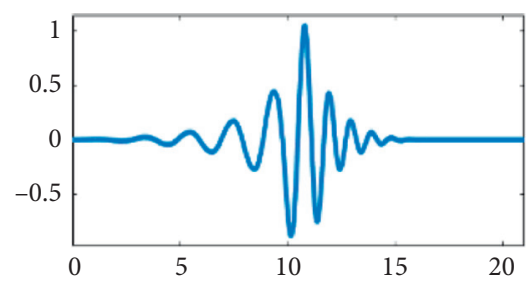

(h)

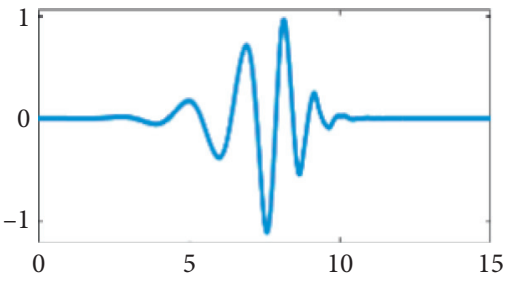

(c)

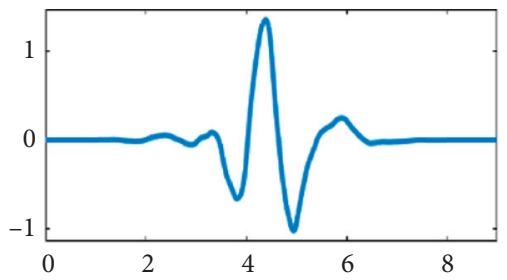

(f)

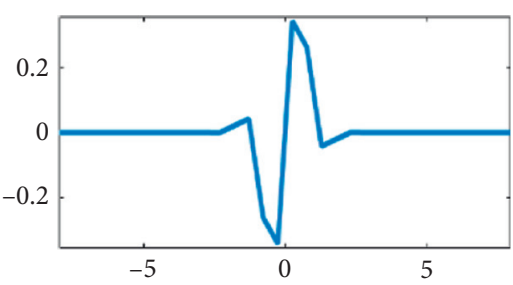

(i)

Figure 6: Wavelet packet basis function: (a) dmey; (b) Haar; (c) db8; (d) db10; (e) sym3; (f) sym5; (g) coif3; (h) fk22; (i) cmor1-1.5.

$$
Y=\left[y_{1}, y_{2}, y_{3}, \ldots, y_{m}\right]
$$

We calculated the number of intermediate layers in Figure 14 from the following equation:

$$
p=\sqrt{n+m}+l
$$

where $n$ represents the number of network input nodes. The extracted sEMG signal feature vector is $n=12$, where $m$ represents the six motion intention output prediction results 


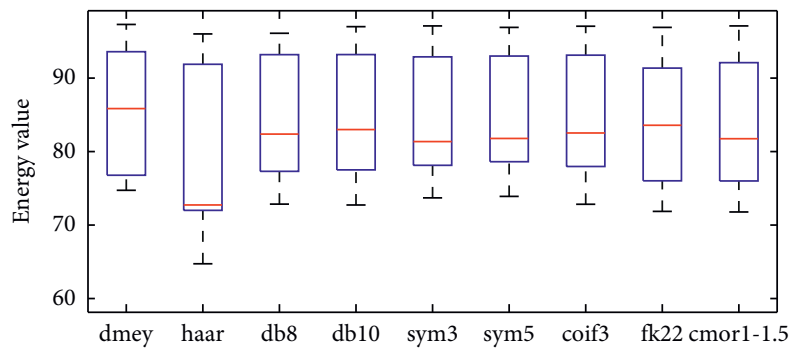

Figure 7: Nine energy box graphs of the wavelet packet basis functions are drawn in six motion intentions.

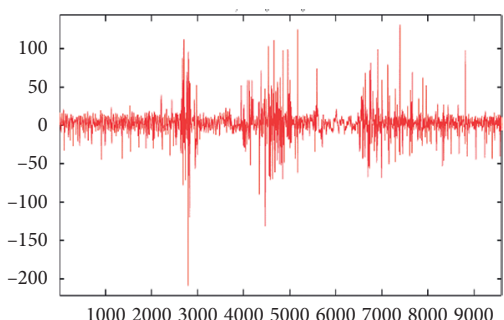

(a)

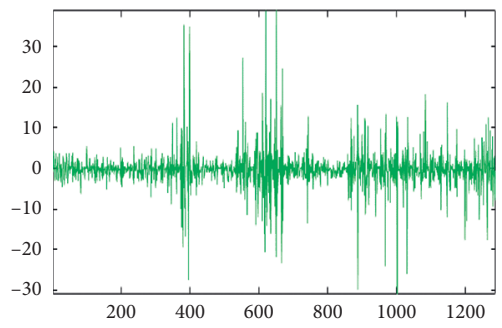

(d)

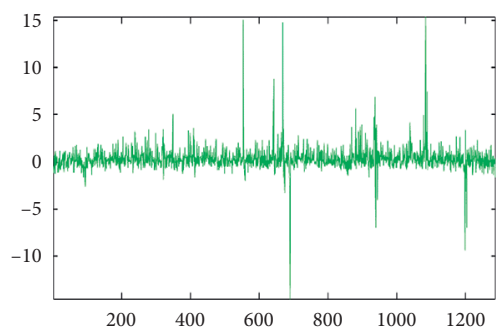

(g)

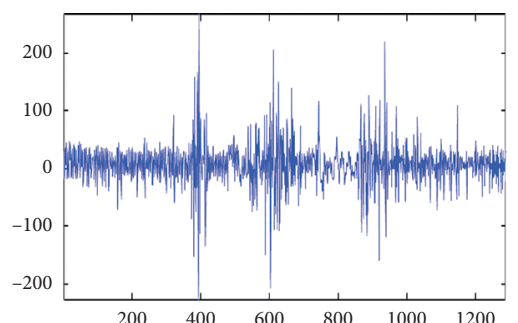

(b)

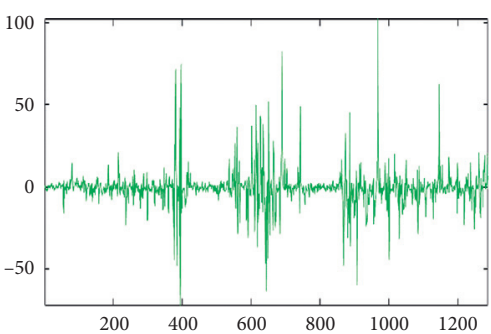

(e)

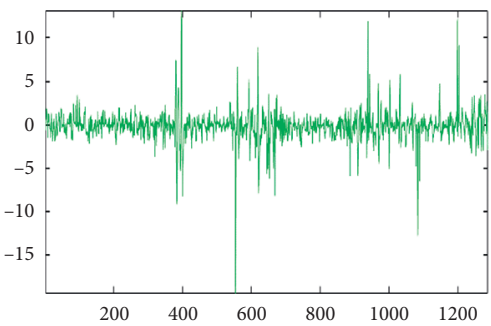

(h)

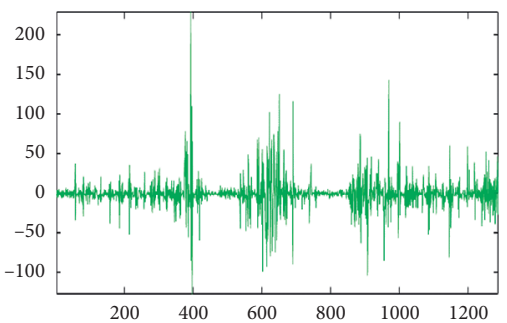

(c)

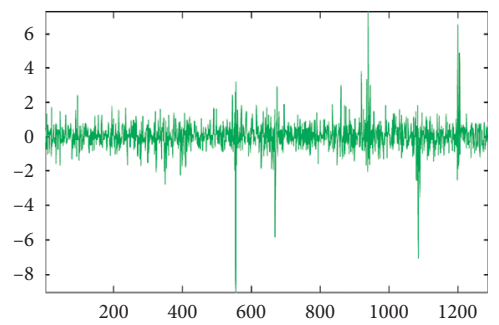

(f)

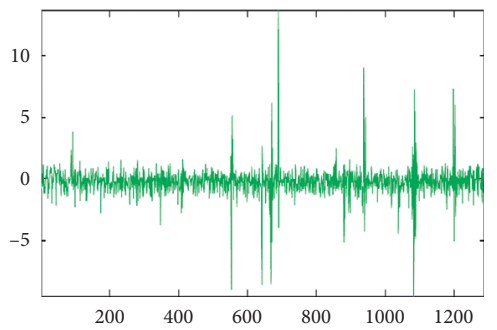

(i)

Figure 8: Using WPT to decompose the sEMG signal of the gastrocnemius: (a) $S$; (b) $\mathrm{AAA}_{3}$; (c) $\mathrm{DAA}_{3}$; (d) $\mathrm{ADA}_{3}$; (e) $\mathrm{DDA}_{3}$; (f) $\mathrm{AAD}_{3}$; (g) $\mathrm{DAD}_{3}$; (h) $\mathrm{ADD}_{3}$; (i) $\mathrm{DDD}_{3}$.

of the lower limbs and $l$ is a constant greater than 1 and less than 10 .

We used the UKF filtering algorithm to correct the threelayer neural network connection weights. The input to the neural network is $X$, constructing the state equation of the UKF filtering algorithm with the weight of the neural network. The output of the neural network is taken as its measurement equation. Therefore, the state equation and measurement equation of the UKF algorithm are as follows:

$$
\begin{aligned}
w_{k+1} & =w_{k}+\omega_{k}, \\
y_{k} & =f_{k}\left(w_{k}, x_{k}\right)+v_{k},
\end{aligned}
$$

where $w_{k}$ is the weight, $x_{k}$ is the input vector, $y_{k}$ is the output of the neural network, and $f_{k}$ is the transfer function of the neural network. And $\omega_{k}$ and $v_{k}$ are the input noise and measurement noise of the neural network. All obey the mean value of 0 , and the variance is the normal distribution of $R^{W}$ and $R^{V}$.

\section{Experiment Results and Discussion}

In the process of extracting the features of the sEMG signal on the lower limb surface, we used the WPT algorithm and selected the best mother wave to extract the sEMG signal features. We analyzed the obtained distribution of features 
TABLE 1: Wavelet packet energy eigenvalues.

\begin{tabular}{|c|c|c|c|c|c|c|c|c|c|}
\hline Motion & Channel & $\mathrm{AAA}_{3}$ & $\mathrm{DAA}_{3}$ & $\mathrm{ADA}_{3}$ & $\mathrm{DDA}_{3}$ & $\mathrm{AAD}_{3}$ & $\mathrm{DAD}_{3}$ & $\mathrm{ADD}_{3}$ & $\mathrm{DDD}_{3}$ \\
\hline $\mathrm{HW}$ & CH.A & 81 & 13 & 0.5 & 3.5 & 0.1 & 0.1 & 0.2 & 0.2 \\
\hline $\mathrm{CO}$ & CH.A & 59 & 27 & 2.4 & 8.6 & 0.1 & 0.4 & 0.8 & 1.2 \\
\hline SU & CH.A & 90 & 7.3 & 0.5 & 1.5 & 0.1 & 0.1 & 0.2 & 0.2 \\
\hline DS & CH.A & 88 & 8.9 & 0.4 & 1.2 & 0.1 & 0.1 & 0.2 & 0.1 \\
\hline GU & CH.A & 91 & 6.5 & 0.4 & 1.1 & 0.1 & 0.1 & 0.1 & 0.1 \\
\hline SR & CH.A & 91 & 2.9 & 1.5 & 1.9 & 0.3 & 0.4 & 1.0 & 0.7 \\
\hline HW & СH.B & 74 & 19 & 0.9 & 4.4 & 0.1 & 0.1 & 0.1 & 0.3 \\
\hline $\mathrm{CO}$ & CH.B & 90 & 7.6 & 0.3 & 1.2 & 0.1 & 0.0 & 0.1 & 0.1 \\
\hline SU & CH.B & 83 & 12 & 0.6 & 2.9 & 0.1 & 0.1 & 0.1 & 0.2 \\
\hline DS & CH.B & 76. & 18 & 0.8 & 3.9 & 0.1 & 0.1 & 0.2 & 0.2 \\
\hline GU & CH.B & 78 & 14 & 0.5 & 5.3 & 0.1 & 0.2 & 0.1 & 0.2 \\
\hline SR & CH.B & 68 & 17 & 2.6 & 8.5 & 0.3 & 0.4 & 1.2 & 0.9 \\
\hline HW & CH.C & 64 & 22 & 1.9 & 9.8 & 0.1 & 0.2 & 0.6 & 0.9 \\
\hline $\mathrm{CO}$ & CH.C & 89 & 7.2 & 0.6 & 1.7 & 0.1 & 0.1 & 0.2 & 0.4 \\
\hline SU & CH.C & 69 & 22 & 1.3 & 5.6 & 0.0 & 0.2 & 0.5 & 0.5 \\
\hline DS & CH.C & 75 & 15 & 1.3 & 6.3 & 0.0 & 0.1 & 0.3 & 0.3 \\
\hline $\mathrm{GU}$ & CH.C & 68 & 23 & 1.3 & 5.3 & 0.0 & 0.1 & 0.3 & 0.3 \\
\hline SR & CH.C & 86 & 5.8 & 1.8 & 2.8 & 0.6 & 0.4 & 0.9 & 0.6 \\
\hline
\end{tabular}

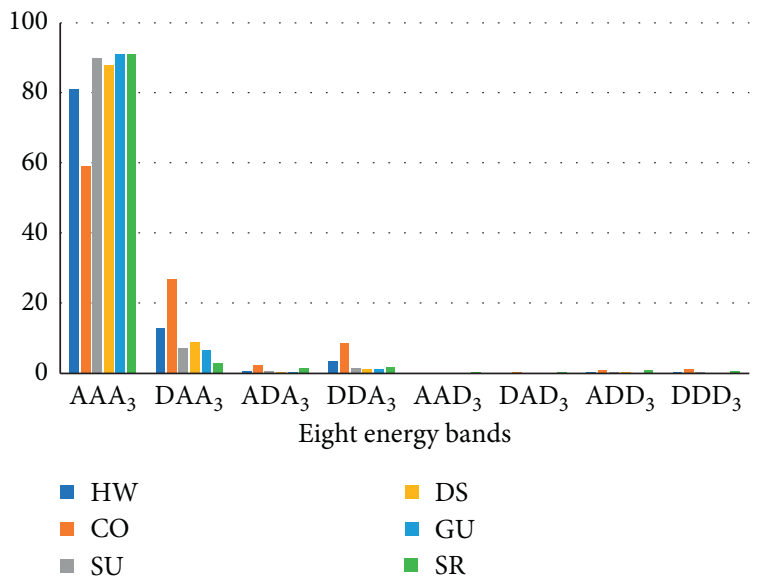

(a)

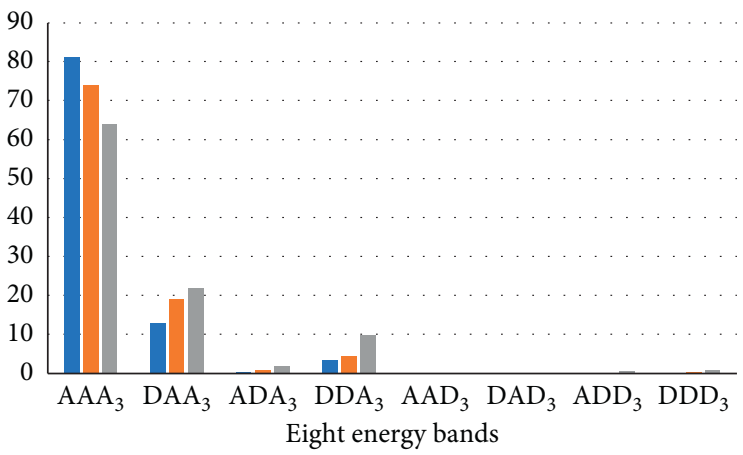

- CH.A

- CH.B

- CH.C

FIgURE 9: Energy feature distribution: (a) energy distribution of different motion intentions in CH.A channel; (b) energy distribution of different channels in the HW mode.

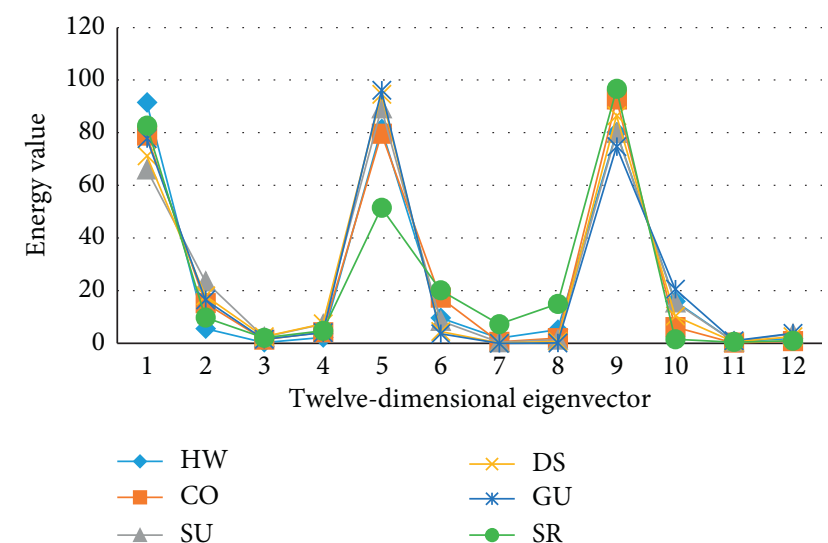

FIGURE 10: sEMG signal eigenvector model map of six motion intentions of lower limbs. and determined the eigenvectors as $\mathrm{AAA}_{3}, \mathrm{DAA}_{3}, \mathrm{ADA}_{3}$, and $\mathrm{DDA}_{3}$. We established a feature model for different motion intentions, as shown in Figure 10. Considering that during the motion of the lower limbs of the human body, the sEMG signal will be affected by noise. The extracted features containing noise interference are different from the features in the motion, as shown in Figure 12. Therefore, this paper proposed a sliding window difference averaging filtering method, verifying the feature model to extract stable eigenvectors, as shown in Figure 13.

In the recognition of lower limb motion intention, this paper designed the UKFNN algorithm, using the UKF filtering algorithm to establish a neural network state-space model and measurement model, optimizing the neural network weight vector, and solving the problem that the neural network has slow convergence speed and poor 


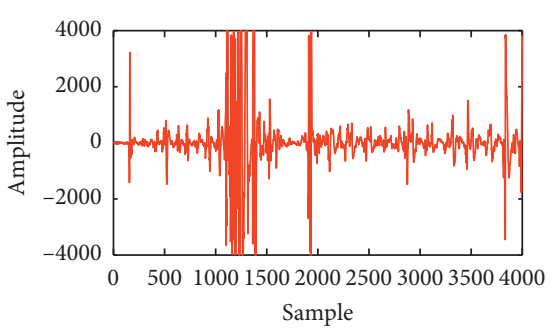

(a)

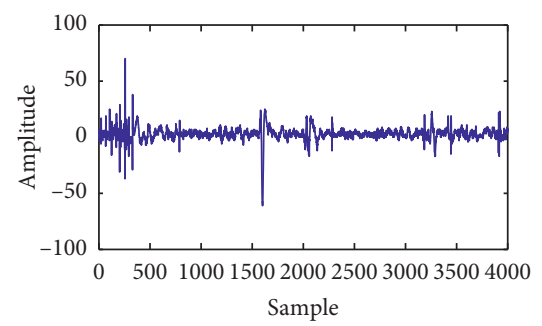

(d)

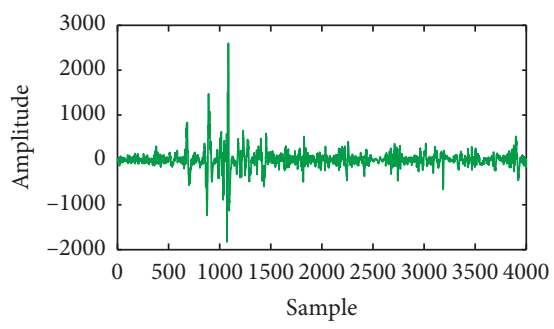

(g)

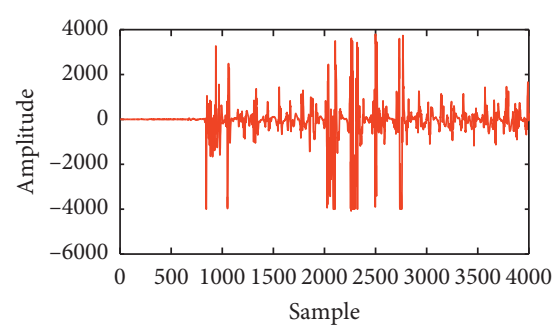

(b)

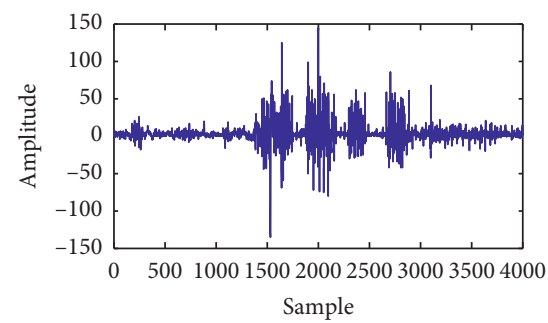

(e)

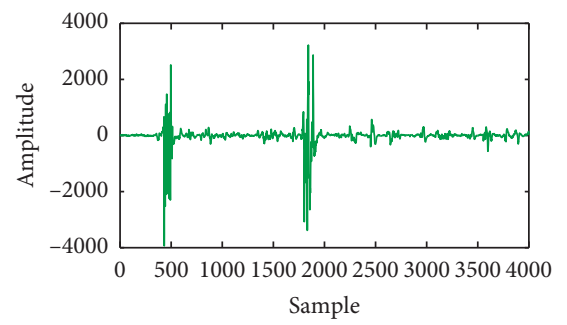

(h)

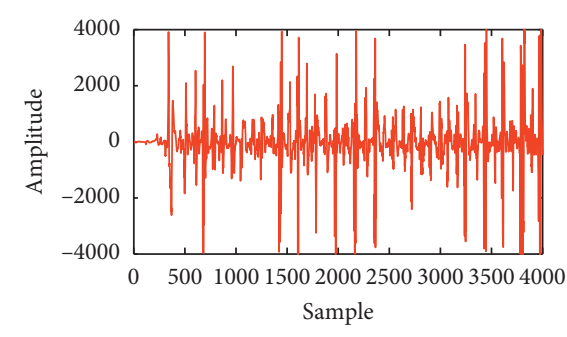

(c)

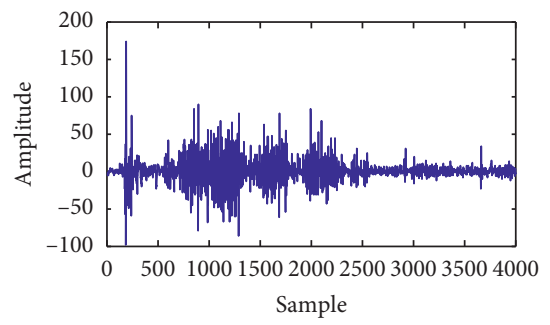

(f)

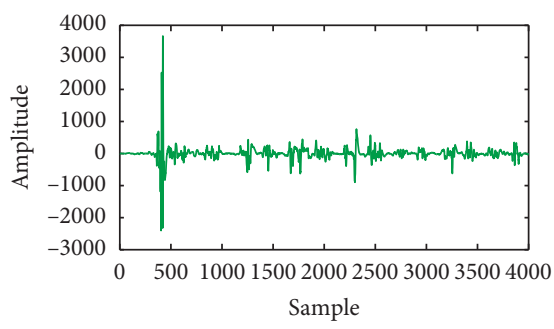

(i)

FIGURE 11: SEMG signal including three kinds of interference noise: (a) SEMG signal of semitendinosus with friction interference; (b) SEMG signal of lateral femoris with friction interference; (c) SEMG signal of medial gastrocnemius with friction interference; (d) SEMG signal of semitendinosus with sweat interference; (e) SEMG signal of lateral femoris with sweat interference; (f) SEMG signal of medial gastrocnemius with sweat interference; (g) SEMG signal of semitendinosus including friction and sweat interference; (h) SEMG signal of lateral femoris including friction and sweat interference; (i) SEMG signal of medial gastrocnemius including friction and sweat interference.

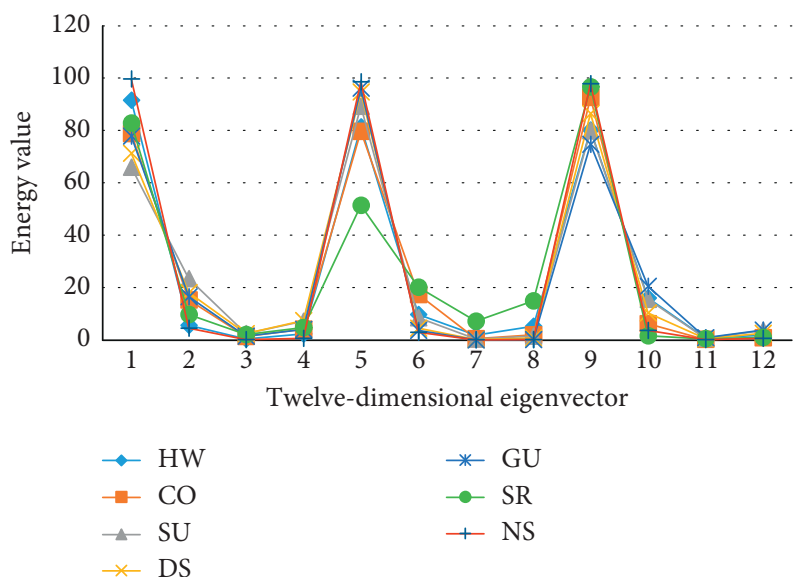

(a)

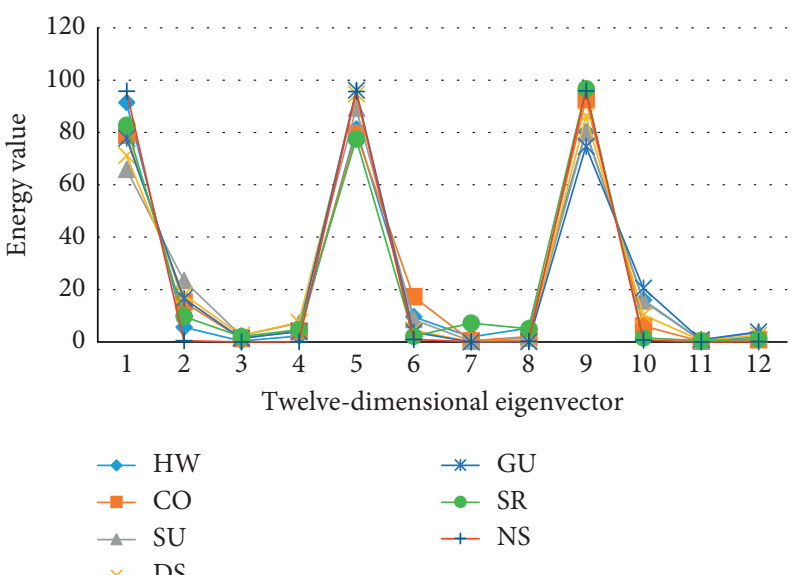

(b)

Figure 12: Continued. 


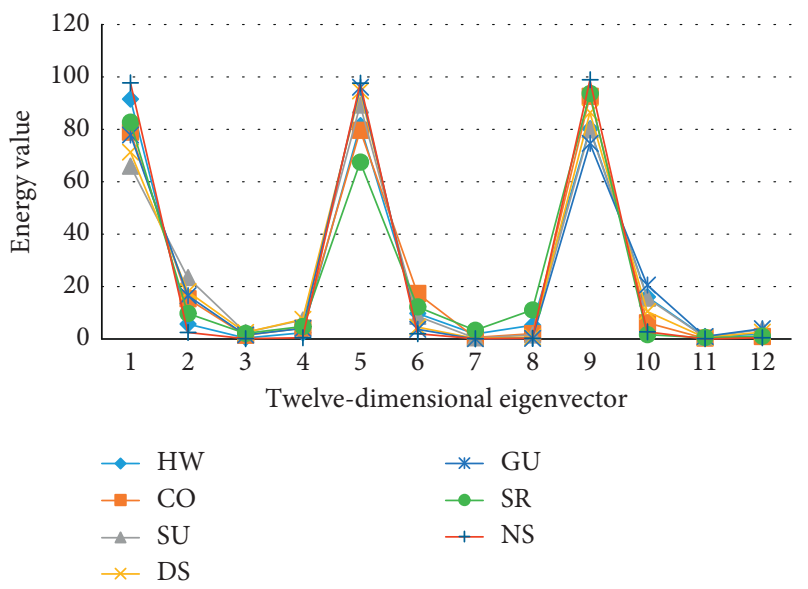

(c)

FIGURE 12: Energy eigenvalue model containing (a) friction noise interference, (b) sweat noise interference, and (c) friction and sweat noise interference. NS stands for noise interference.

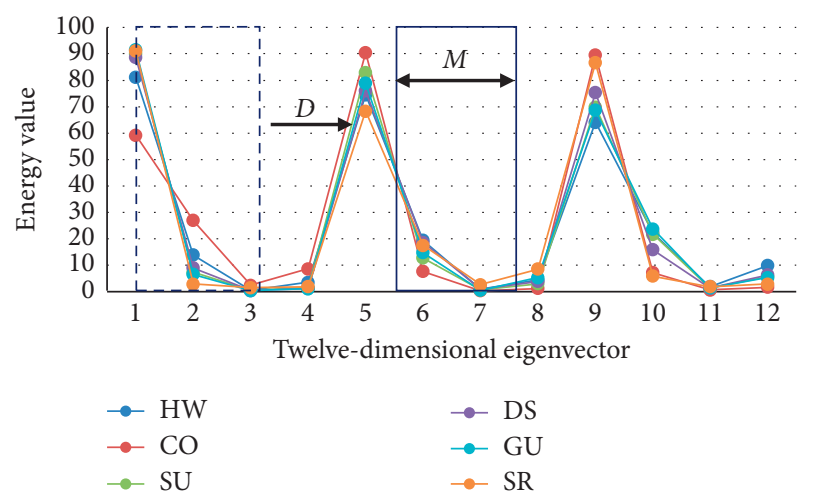

FIGURE 13: Extraction of eigenvalues by difference average filtering of sliding window.

TABLE 2: Sliding window difference average calculation analysis result.

\begin{tabular}{lcccc}
\hline Motion mode & Window 1 & Window 2 & Window 3 & $\begin{array}{c}\text { Difference } \\
\text { average }\end{array}$ \\
\hline HW & 90 & 79 & 79 & 82 \\
CO & 77 & 79 & 92 & 82 \\
SU & 63 & 89 & 79 & 78 \\
DS & 69 & 94 & 86 & 83 \\
GU & 76 & 96 & 74 & 82 \\
SR & 80 & 44 & 90 & 70 \\
\hline
\end{tabular}

adaptability to noise interference in the model training process.

This paper constructed a 3-layer neural network, as shown in Figure 14. The input is a twelve-dimensional eigenvector, and the output is the predicted result of six motion intentions. The transfer function selected by the hidden layer is a nonlinear unipolar excitation function as Sigmoi $d$. The output layer transfer function selected a linear function as Purelin, which can output an arbitrary value. The number of middle layer neurons is calculated by
TABLE 3: Noise interference energy difference average calculation analysis result.

\begin{tabular}{lcccc}
\hline Noise type & Window 1 & Window 2 & Window 3 & $\begin{array}{c}\text { Difference } \\
\text { average }\end{array}$ \\
\hline Interference 1 & 89 & 94 & 91 & 92 \\
Interference 2 & 88 & 92 & 93 & 91 \\
Interference 3 & 96 & 97 & 95 & 96 \\
\hline
\end{tabular}

Interference 1, sweating; interference 2, friction; interference 3, friction and sweating.

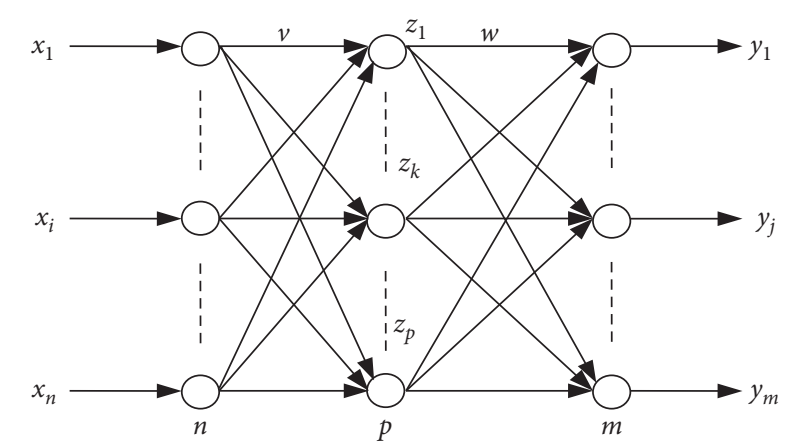

FIgURE 14: Three-layer UKF neural network structure.

formula (7) to be $5 \leq p \leq 14$. Then, we verified through experiments that the number of middle-tier nodes is 14 . The dimension of the UKFNN algorithm weight variable is 183 .

We collected 600 sets of samples and divided that into training sets and testing sets, respectively, 480 sets and 120 sets. Both training sets and testing sets contain six motion data for five subjects. We trained the model with 480 sets of data. We selected each subject's own 24 testing sets to verify the accuracy of six lower extremity motion intention. We verified a total of five subjects. The lower limb motion recognition accuracy was defined as the ratio of the number of correct recognition results of each move to the 
number of corresponding motion in the testing sets. The recognition accuracy is shown in Figure 15. The average accuracy of the UKFNN model is $94.83 \%$. The average accuracy of the BPNN model is $90.8 \%$. The minimum accuracy of the UKFNN model is $91.33 \%$, and the accuracy of the BPNN model is $88.16 \%$. It is concluded that the average accuracy of the UKFNN model is higher than the BPNN model.

Also, robustness is one of the important parameters to evaluate the performance of the algorithm, so we calculated the standard deviation based on the results of Figure 15 as an evaluation of robustness. As can be seen from Table 4, UKFNN is more robust than BPNN.

To graphically describe the distribution of the accuracy, a box plot is drawn, as shown in Figure 16. According to Figure 16, the accuracy rate of the UKFNN is higher than that of the BPNN, and the dispersion of the UKFNN is smaller than that of the BPNN.

Tables 5 and 6 show the results of motion intention recognition accuracy of the UKFNN model and the BPNN model for five subjects with six lower limb motion intentions.

In the UKFNN algorithm, the highest average accuracy is the go-upstairs (GU) motion, with $97.8 \%$. The lowest average accuracy is stop-rest (SR) motion, with $88.8 \%$. In the BPNN algorithm, the highest average accuracy is the go-upstairs (GU) motion, with $94.6 \%$. The lowest average accuracy is horizontal walking (HW) motion, with $87 \%$. Experiments show that the accuracy of the UKFNN model is more stable than the BPNN model.

Besides, the anti-interference of the model is an important factor affecting the control performance of the lower extremity exoskeleton. Considering that the sEMG signal is susceptible to noise interference, we tested the collected noise sEMG signals (interference 1, interference 2, and interference 3) and verified the ability of the UKFNN model to track sEMG signals. As shown in Figure 17, under different interference conditions, the UKFNN model can still track the change of features in real-time.

We calculated the root mean square error of the model as metrics to evaluate the reliability of the model, as shown in Table 7. It is proved that the UKFNN model has a stronger anti-interference ability than the BPNN model.

We found that different numbers of sEMG signal feature samples affect the motion intention recognition accuracy and real-time. We collected another 600 sets of data for testing and using different numbers of testing samples, namely, 100, 200, 400, and 600 samples, to test the UKFNN model and the BPNN model. The result is shown in Table 8.

It is proved that the samples increase and the accuracy of the BPNN and the UKFNN model will also increase. In the process of sample size change, the UKFNN model has higher accuracy, stability, and real-time performance than the BPNN model.

When the user is wearing the lower extremity exoskeleton, it is necessary to avoid the wrong recognition of human motion intention by the lower extremity exoskeleton robots. The confusion matrix is an important evaluation method of model accuracy and reliability. It can count the

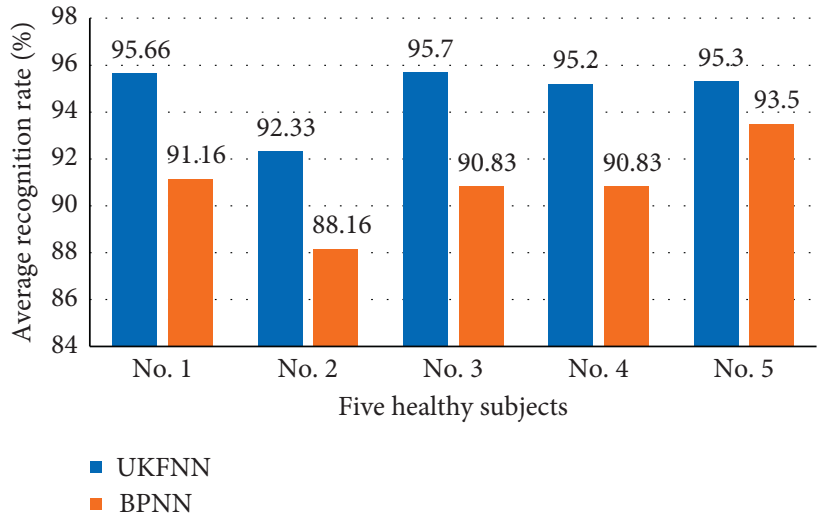

FIGURE 15: UKFNN model and BPNN model accuracy results.

TABLE 4: UKFNN model for each motion accuracy result.

\begin{tabular}{lcc}
\hline Recognition algorithm & UKFNN & BPNN \\
\hline Average accuracy rate (\%) & 94.83 & 90.8 \\
Standard deviation & 1.26 & 1.69 \\
\hline
\end{tabular}

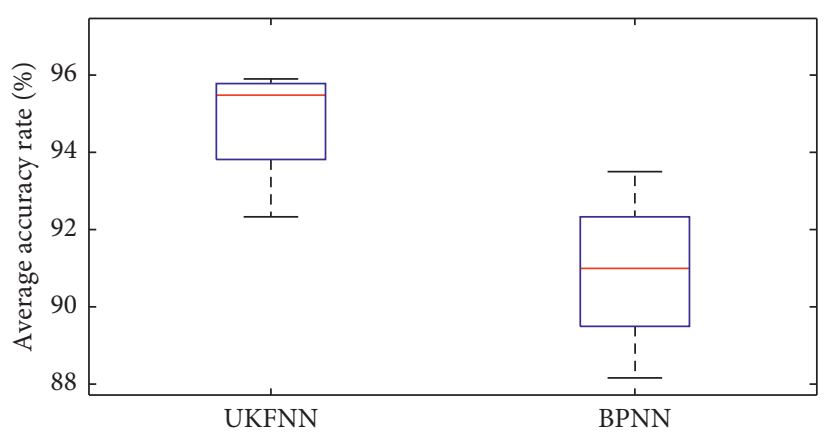

Figure 16: Box plot of UKFNN and BPNN.

TABLE 5: UKFNN model for each motion accuracy result.

\begin{tabular}{lcccccc}
\hline \multirow{2}{*}{ Subject } & \multicolumn{6}{c}{ Motion intention (\%) } \\
& HW & CO & SU & GU & DS & SR \\
\hline 1 & 96 & 99 & 92 & 98 & 99 & 90 \\
2 & 83 & 90 & 96 & 99 & 96 & 90 \\
3 & 98 & 99 & 98 & 97 & 95 & 86 \\
4 & 93 & 96 & 98 & 96 & 98 & 90 \\
5 & 96 & 95 & 100 & 98 & 96 & 88 \\
AC (\%) & 93.2 & 95.8 & 96.6 & 97.8 & 96.8 & 88.8 \\
\hline
\end{tabular}

TABLE 6: BPNN model for each motion accuracy result.

\begin{tabular}{lcccccc}
\hline \multirow{2}{*}{ Subject } & \multicolumn{6}{c}{ Motion intention (\%) } \\
& HW & CO & SU & GU & DS & SR \\
\hline 1 & 86 & 95 & 93 & 99 & 86 & 88 \\
2 & 78 & 91 & 90 & 95 & 83 & 92 \\
3 & 91 & 92 & 95 & 93 & 86 & 88 \\
4 & 86 & 87 & 100 & 90 & 92 & 90 \\
5 & 94 & 93 & 95 & 87 & 94 & 98 \\
AC (\%) & 87 & 91.6 & 94.6 & 92.8 & 88.2 & 91.2 \\
\hline
\end{tabular}




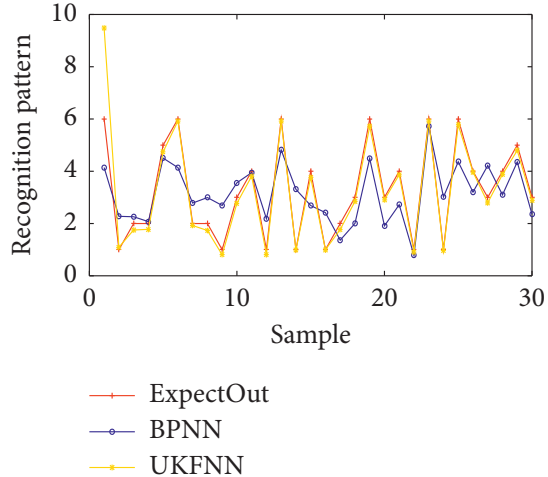

(a)
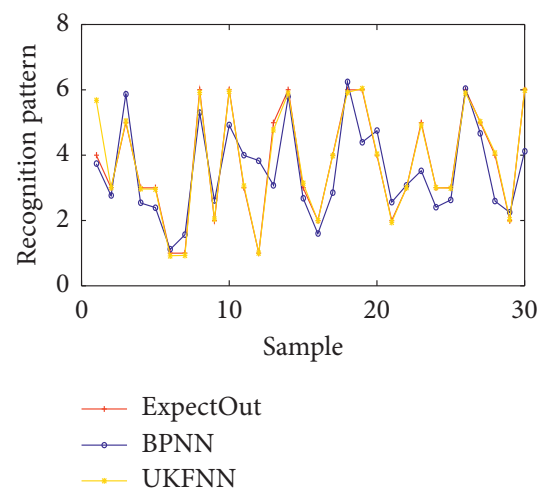

(d)

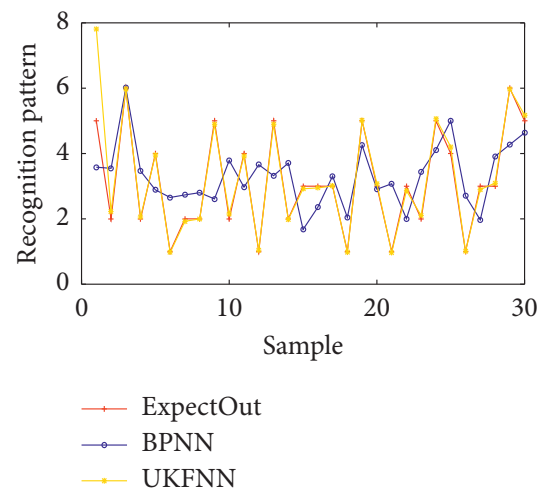

(g)

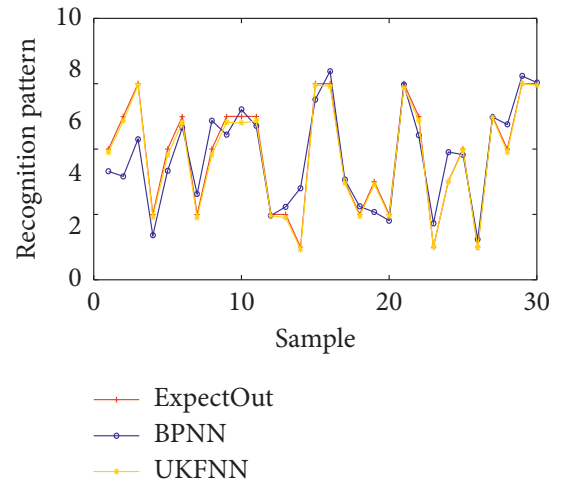

(b)

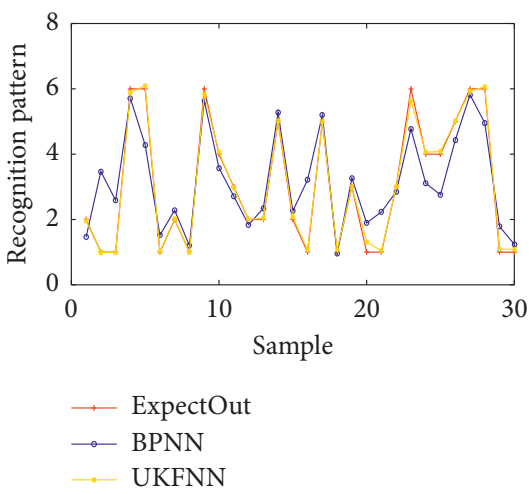

(e)

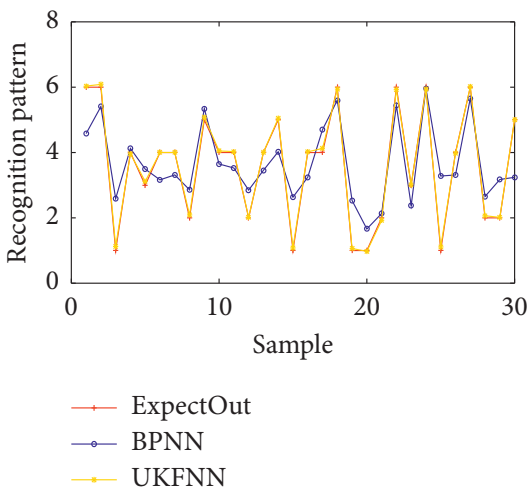

(h)

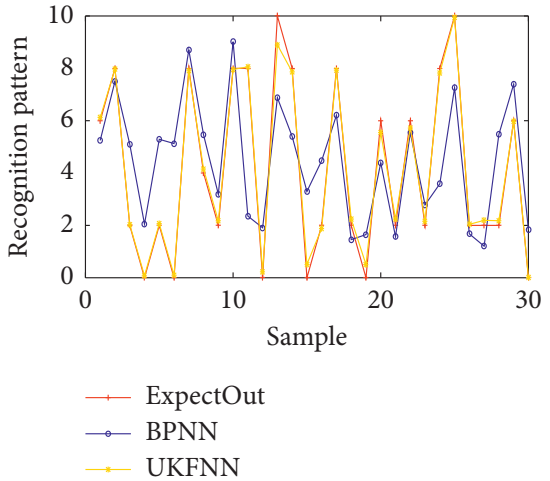

(c)

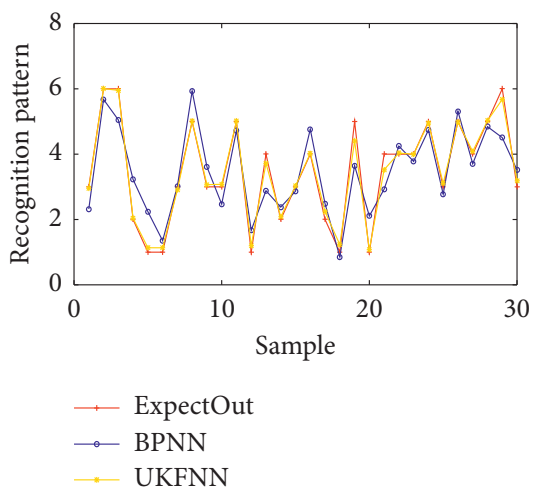

(f)

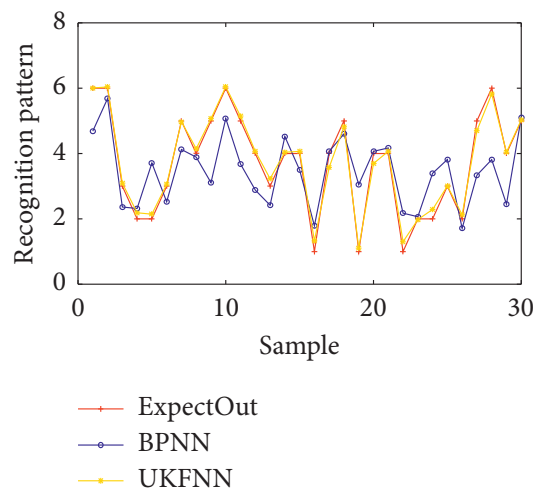

(i)

FIGURE 17: UKFNN model and BPNN model recognize the result. UKFNN model and BPNN model interference test: (a) subject 1 sweating interference test; (b) subject 1 frictional interference test; (c) subject 1 sweating and frictional interference test; (d) subject 2 sweating interference test; (e) subject 2 frictional interference test; (f) subject 2 sweating and frictional interference test; (g) subject 3 sweating interference test; (h) subject 3 frictional interference test; (i) subject 3 sweating and friction interference test.

number of errors in the model recognition results and the correct number and also can analyze the correlation between different motion intentions of the lower limbs. In this paper, 400 sets of testing samples are selected to calculate the confusion matrix of six lower limb motion intentions and verified the accuracy of the method. Table 9 is the confusion matrix of the UKFNN model. The stop-rest (SR) motion intention has the lowest accuracy $(88.8 \%)$. It is easy to misjudge the down-stairs (DS) motion intention, and the false-positive rate is $5.1 \%$. Table 10 is the confusion matrix of the BPNN model. The horizontal walking (HW) motion intention has the lowest average accuracy (87\%). It is easy to misjudge the motion intention of go-upstairs (GU), and the false-positive rate is $6.5 \%$.

We selected 600 testing sets to verify the sensitivity of the model. Table 11 shows the sensitivity metrics of the UKFNN model and the BPNN model. The UKFNN model has a higher sensitivity. It is proved that the UKFNN model has a lower false-positive rate, and accuracy and reliability are better than the BPNN model.

We used three myoelectric sensors to identify the six motion intentions of the lower extremities. Also, we 
TABLE 7: RMS error statistics of UKFNN and BPNN interference tests.

\begin{tabular}{llcr}
\hline Type of interference & & \multicolumn{2}{c}{ Root mean square error statistics } \\
& & BPNN & 0.17 \\
Subject 1 & Interference 1 & 0.21 & 0.13 \\
& Interference 2 & 0.18 & 0.08 \\
\hline \multirow{3}{*}{ Subject 2 } & Interference 3 & 0.12 & 0.24 \\
& Interference 1 & 0.35 & 0.16 \\
\hline \multirow{3}{*}{ Subject 3 } & Interference 2 & 0.29 & 0.13 \\
& Interference 3 & 0.18 & 0.14 \\
& Interference 1 & 0.25 & 0.16 \\
& Interference 2 & 0.17 & 0.03 \\
\hline
\end{tabular}

TABLE 8: Performance test of UKFNN and BPNN models with different sample sizes.

\begin{tabular}{lcccc}
\hline \multirow{2}{*}{ Recognition algorithm } & \multicolumn{3}{c}{ Metrics } \\
& Number of samples & Recognition accuracy (\%) & Root mean square error & Average running time (S) \\
\hline \multirow{3}{*}{ UKFNN } & 100 & 92.17 & 0.17 & 0.194 \\
& 200 & 93.45 & 0.16 & 0.239 \\
\hline & 400 & 91.75 & 0.08 & 0.924 \\
BPNN & 600 & 94.83 & 0.04 & 1.041 \\
& 100 & 81.7 & 0.21 & 0.18 \\
\hline
\end{tabular}

TABLE 9: UKFNN model confusion matrix.

\begin{tabular}{|c|c|c|c|c|c|c|}
\hline \multirow{2}{*}{ Expected motion } & \multicolumn{6}{|c|}{ Real motion } \\
\hline & HW & $\mathrm{CO}$ & SU & GU & DS & SR \\
\hline HW (\%) & 93.2 & - & - & 4.3 & - & 2.5 \\
\hline $\mathrm{CO}(\%)$ & - & 95.8 & - & 4.1 & - & 0.1 \\
\hline SU (\%) & - & - & 96.6 & 3.4 & - & - \\
\hline GU (\%) & - & 1.9 & - & 97.8 & 0.3 & - \\
\hline DS (\%) & - & - & 2.1 & - & 96.8 & 1.1 \\
\hline SR $(\%)$ & 0.3 & 2.1 & 3.7 & - & 5.1 & 88.8 \\
\hline
\end{tabular}

TABLE 10: BPNN model confusion matrix.

\begin{tabular}{|c|c|c|c|c|c|c|}
\hline \multirow{2}{*}{ Expected motion } & \multicolumn{6}{|c|}{ Real motion } \\
\hline & HW & $\mathrm{CO}$ & SU & GU & DS & SR \\
\hline HW (\%) & 87 & - & 3.2 & 6.5 & 2.4 & 0.9 \\
\hline $\mathrm{CO}(\%)$ & - & 91.6 & - & 5.4 & 2.6 & 0.4 \\
\hline SU (\%) & - & - & 94.6 & 0.6 & 3.6 & 1.2 \\
\hline GU (\%) & - & 4.9 & 0.7 & 92.8 & 1.6 & - \\
\hline DS (\%) & - & 3.3 & 5.5 & 0.9 & 88.2 & 2.1 \\
\hline SR (\%) & - & 0.3 & 4.1 & - & 4.4 & 91.2 \\
\hline
\end{tabular}

TABLE 11: Sensitivity metrics of the UKFNN model and the BPNN model.

\begin{tabular}{lccccc}
\hline \multirow{2}{*}{ Recognition algorithm } & \multicolumn{4}{c}{ Sensitivity (\%) } \\
& HW & CO & SU & GU & DS \\
\hline UKFNN & 92 & 95 & 97 & 98 & 95 \\
BPNN & 89 & 92 & 91 & 93 & 90 \\
\hline
\end{tabular}


TABLE 12: This paper proposes a comparison of lower limb motion intention recognition algorithms with other recognition methods.

\begin{tabular}{|c|c|c|c|c|c|}
\hline Author & $\begin{array}{c}\text { Number of myoelectric } \\
\text { sensors }\end{array}$ & $\begin{array}{l}\text { Types of } \\
\text { motion }\end{array}$ & Recognition methods & $\begin{array}{c}\text { Recognition accuracy } \\
(\%)\end{array}$ & References \\
\hline Al-Quraishi et al. & 4 & 4 & LDA & 94.36 & [7] \\
\hline Coelho and Lima & 8 & 7 & $\begin{array}{l}\text { Fractal dimension } \\
\text { estimation }\end{array}$ & 95.87 & [9] \\
\hline $\begin{array}{l}\text { Toledo-Pérez } \\
\text { et al. }\end{array}$ & 4 & 3 & PCA and SVM & 97.66 & {$[10]$} \\
\hline Tang et al. & 3 & 3 & DWT and BPNN & 96.33 & {$[11]$} \\
\hline Ai et al. & 4 & 5 & DWT and LDA & 94.41 & {$[14]$} \\
\hline Zhang et al. & 1 & 4 & SVM & 91.85 & [16] \\
\hline Propose method & 3 & 6 & WPT and UKFNN & 94.83 & This work \\
\hline
\end{tabular}

compared the results of previous studies to demonstrate the advancement of our recognition algorithm, as shown in Table 12. In these studies, most researchers used more sEMG sensors to identify more lower extremity motions. It can be seen from Table 12 that at least four muscle sEMG signals need to be acquired to identify five lower limb motion intentions. However, we only need three sensors to identify six lower limb motion intentions, and the accuracy reaches $94.83 \%$.

\section{Conclusion}

We proposed a lower limb motion recognition method based on improved WPT and UKFNN. We analyzed the distribution of features and established a feature model. And we proposed a sliding window difference averaging filtering method to verify the eigenvalues and suppressed the interference of noise. Then, we used UKF filtering to optimize the neural network weights to improve the adaptability and accuracy of the recognition model, and we used the UKFNN model to identify the six motion intentions of the lower limbs. The results showed that the method has achieved good results and the average accuracy was $94.83 \%$. Through the noise interference tests, it was verified that the model has the anti-interference ability. Also, under the fluctuation of the sample size, the UKFNN model has achieved better results in recognition accuracy and real-time performance. Also, the confusion matrix calculated that the UFKNN model has a low falsepositive rate and the performance was better than the BPNN model. It showed that the method was advanced, reliable, and practical. Therefore, our proposed new method was suitable for the recognition of complex motion intentions of lower limbs. It is beneficial to realize the wide application of the exoskeleton robots to assist the lower limb motion. In the next step, the paper will increase the types of lower limb motion intention recognition, improving the recognition accuracy of lower limb motion intention.

\section{Data Availability}

The data used to support the findings of this study are available from the corresponding author upon request.

\section{Conflicts of Interest}

The authors declare that there are no conflicts of interest.

\section{Authors' Contributions}

P.Q. did conceptualization and methodology. X.S. administered the project.

\section{Acknowledgments}

Special thanks are due to Jiaqing Zhu and Maqiang Zhai for the support provided in preparing the experiments. This work was supported by Projects U1613226 and U1813217 supported by NSFC, China, and Project 2019-INT009 from the Shenzhen Institute of Artificial Intelligence and Robotics for Society.

\section{References}

[1] P. Phukpattaranont, S. Thongpanja, K. Anam, A. Al-Jumaily, and C. Limsakul, "Evaluation of feature extraction techniques and classifiers for finger movement recognition using surface electromyography signal," Medical \& Biological Engineering \& Computing, vol. 56, no. 12, pp. 2259-2271, 2018.

[2] X. Yang, H. She, H. Lu, T. Fukuda, and Y. Shen, "State of the art: bipedal robots for lower limb rehabilitation," Applied Sciences, vol. 7, no. 11, p. 1182, 2017.

[3] B. Gao, C. Wei, H. Ma, S. Yang, X. Ma, and S. Zhang, "Realtime evaluation of the signal processing of sEMG used in limb exoskeleton rehabilitation system," Applied Bionics and Biomechanics, vol. 2018, Article ID 1391032, 6 pages, 2018.

[4] L. Grazi, S. Crea, A. Parri et al., "Gastrocnemius myoelectric control of a robotic hip exoskeleton," in Proceedings of the 2015 37th Annual International Conference of the IEEE Engineering in Medicine and Biology Society (EMBC), pp. 3881-3884, Milan, Italy, August 2015.

[5] A. von Glinski, E. Yilmaz, S. Mrotzek et al., "Effectiveness of an on-body lifting aid (HAL for care support) to reduce lower back muscle activity during repetitive lifting tasks," Journal of Clinical Neuroscience, vol. 63, pp. 249-255, 2019.

[6] F. Zhang, Z.-G. Hou, L. Cheng et al., "iLeg-a lower limb rehabilitation robot: a proof of concept," IEEE Transactions on Human-Machine Systems, vol. 46, no. 5, pp. 761-768, 2016.

[7] M. S. Al-Quraishi, A. J. Ishak, S. A. Ahmad et al., "Classification of ankle joint motions based on surface electromyography signals for rehabilitation robot applications," Medical 
\& Biological Engineering \& Computing, vol. 55, no. 5, pp. 747-758, 2017.

[8] B. Xiong, N. Zeng, H. Li et al., "Intelligent prediction of human lower extremity joint moment: an artificial neural network approach," IEEE Access, vol. 7, pp. 29973-29980, 2019.

[9] A. L. Coelho and C. A. M. Lima, "Assessing fractal dimension methods as feature extractors for EMG signal classification," Engineering Applications of Artificial Intelligence, vol. 36, pp. 81-98, 2014.

[10] D. C. Toledo-Pérez, M. A. Martínez-Prado, R. A. GómezLoenzo, W. J. Paredes-García, and J. Rodríguez-Reséndiz, “A study of motion classification of the lower limb based on up to 4-EMG channels," Electronics, vol. 8, no. 3, p. 259, 2019.

[11] G. Tang, H. Wang, and Y. Tian, "sEMG-based estimation of knee joint angles and motion intention recognition," in Proceedings of the 2017 9th International Conference on Intelligent Human-Machine Systems and Cybernetics, pp. 390393, Hangzhou, Zhejiang, China, August 2017.

[12] X. Ma, C. Wang, R. Zhang, and X. Wu, "A real-time gait switching method for lower-limb exoskeleton robot based on sEMG signals," in Proceedings of the International Conference on Cognitive Systems and Signal Processing, pp. 511-523, Beijing, China, November 2019.

[13] Q. Li, Y. Song, and Z. G. Hou, "Estimation of lower limb periodic motions from sEMG using least squares support vector regression," Neural Processing Letters, vol. 41, no. 3, pp. 371-388, 2015.

[14] Q. Ai, Y. Zhang, W. Qi, Q. Liu, and K. Chen, "Research on lower limb motion recognition based on fusion of sEMG and accelerometer signals," Symmetry, vol. 9, no. 8, p. 147, 2017.

[15] C. Tapia, O. Daud, and J. Ruiz-del-Solar, "EMG signal filtering based on independent component analysis and empirical mode decomposition for estimation of motor activation intentions," Journal of Medical and Biological Engineering, vol. 37, no. 1, pp. 140-155, 2017.

[16] Y. Zhang, P. Li, X. Zhu et al., "Extracting time-frequency feature of single-channel vastus medialis EMG signals for knee exercise intention recognition," PLoS One, vol. 12, no. 7, Article ID e0180526, 2017.

[17] R. N. Khushaba, A. H. Al-Timemy, A. Al-Ani, and A. AlJumaily, "A framework of temporal-spatial descriptors-based feature extraction for improved myoelectric intention recognition," IEEE Transmotions on Neural Systems and Rehabilitation Engineering, vol. 25, no. 10, pp. 1821-1831, 2017.

[18] A. Phinyomark, R. N. Khushaba, and E. Scheme, "Feature extrmotion and selection for myoelectric control based on wearable EMG sensors," Sensors, vol. 18, no. 5, p. 1615, 2018.

[19] F. Wang, T. Yin, C. Lei, Y. Zhang, Y. Wang, and J. Liu, "Prediction of lower limb joint angle using sEMG based on GA-GRNN," in Proceedings of the 2015 IEEE International Conference on Cyber Technology in Automation, Control, and Intelligent Systems, pp. 1894-1899, Shenyang, China, June 2015.

[20] Q. Meng, J. Zhang, and X. Yang, "Virtual rehabilitation training system based on surface EMG feature extrmotion and analysis," Journal of Medical Systems, vol. 43, no. 3, p. 48, 2019.

[21] X. Xi, M. Tang, and Z. Luo, "Feature-level fusion of surface electromyography for activity monitoring," Sensors, vol. 18, no. 2, p. 614, 2018.

[22] J. Chen, X. Zhang, Y. Cheng, and N. Xi, "Surface EMG based continuous estimation of human lower limb joint angles by using deep belief networks," Biomedical Signal Processing and Control, vol. 40, pp. 335-342, 2018.
[23] J. Ryu, B.-H. Lee, and D.-H. Kim, "sEMG signal-based lower limb human motion detection using a top and slope feature extraction algorithm," IEEE Signal Processing Letters, vol. 24, no. 7, pp. 929-932, 2016.

[24] E. Gokgoz and A. Subasi, "Comparison of decision tree algorithms for EMG signal classification using DWT," Biomedical Signal Processing and Control, vol. 18, pp. 138-144, 2015.

[25] A. S. U. Doulah, S. A. Fattah, W.-P. Zhu, and M. O. Ahmad, "Wavelet domain feature extraction scheme based on dominant motor unit motion potential of EMG signal for neuromuscular disease classification," IEEE Transactions on Biomedical Circuits and Systems, vol. 8, no. 2, pp. 155-164, 2014.

[26] X. Chen, P. Xie, H. Liu, Y. Song, and Y. Du, "Local band spectral entropy based on wavelet packet applied to surface EMG signals analysis," Entropy, vol. 18, no. 2, p. 41, 2016.

[27] Y. Ji, S. Sun, and H.-B. Xie, "Stationary wavelet-based twodirectional two-dimensional principal component analysis for EMG signal classification," Measurement Science Review, vol. 17, no. 3, pp. 117-124, 2017.

[28] M. Wang, X. A. Wang, C. Peng, S. Zhang, Z. Fan, and Z. Liu, "Research on EMG segmentation algorithm and walking analysis based on signal envelope and integral electrical signal," Photonic Network Communications, vol. 37, no. 2, pp. 195-203, 2019.

[29] J. Wang, L. Wang, S. M. Miran, X. Xi, and A. Xue, "Surface electromyography based estimation of knee joint angle by using correlation dimension of wavelet coefficient," IEEE Access, vol. 7, pp. 60522-60531, 2019.

[30] C. Cui, G.-B. Bian, Z.-G. Hou, J. Zhao, and H. Zhou, "A multimodal framework based on integration of cortical and muscular activities for decoding human intentions about lower limb motions," IEEE Transactions on Biomedical Circuits and Systems, vol. 11, no. 4, pp. 889-899, 2017.

[31] M.-R. Lee, J.-H. Ryu, S.-H. Kim, and D.-H. Kim, "Gait phase recognition based on EMG signal for stairs ascending and stairs descending," Journal of the Institute of Electronics and Information Engineers, vol. 52, no. 3, pp. 181-189, 2015.

[32] N. Nazmi, M. Abdul Rahman, S.-I. Yamamoto, S. Ahmad, H. Zamzuri, and S. Mazlan, "A review of classification techniques of EMG signals during isotonic and isometric contractions," Sensors, vol. 16, no. 8, p. 1304, 2016.

[33] F. Duan, L. Dai, W. Chang, Z. Chen, C. Zhu, and W. Li, "sEMG-based identification of hand motion intention using wavelet neural network combined with discrete wavelet transform," IEEE Transactions on Industrial Electronics, vol. 63, no. 3, pp. 1923-1934, 2016.

[34] Y. M. Aung and A. Al-Jumaily, "Estimation of upper limb joint angle using surface EMG signal," International Journal of Advanced Robotic Systems, vol. 10, no. 10, p. 369, 2013.

[35] L. Zhang, Y. Shi, W. Wang, Y. Chu, and X. Yuan, "Real-time and user-independent feature classification of forearm using EMG signals," Journal of the Society for Information Display, vol. 27, no. 2, pp. 101-107, 2019.

[36] O. Erkaymaz, I. Şenyer, and R. Uzun, "Detection of knee abnormality from surface EMG signals by artificial neural networks," in Proceedings of the 2017 25th Signal Processing and Communications Applications Conference, pp. 1-4, Antalya, Turkey, May 2017.

[37] S. Thongpanja, A. Phinyomark, F. Quaine et al., "Probability density functions of stationary surface EMG signals in noisy environments," IEEE Transactions on Instrumentation and Measurement, vol. 65, no. 7, pp. 1547-1557, 2016. 
[38] Y. Geng, Y. Ouyang, O. W. Samuel et al., "A robust sparse representation based intention recognition approach for myoelectric control," IEEE Access, vol. 6, pp. 38326-38335, 2018.

[39] Z. Deng, L. Yin, B. Huo, and Y. Xia, “Adaptive robust unscented Kalman filter via fading factor and maximum correntropy criterion," Sensors, vol. 18, no. 8, p. 2406, 2018.

[40] J. Stauch and M. Jah, "Unscented Schmidt-Kalman filter algorithm," Journal of Guidance, Control, and Dynamics, vol. 38, no. 1, pp. 117-123, 2015.

[41] Z. Li, Y. Wang, and Z. Liu, "Unscented Kalman filter-trained neural networks for slip model prediction," PLoS One, vol. 11, no. 7, Article ID e0158492, 2016.

[42] L. Tong, F. Zhang, Z.-G. Hou, W. Wang, and L. Peng, "BPAR-based human joint angle estimation using multi-channel sEMG," Biomedical Signal Processing and Control, vol. 10, pp. 1335-1342, 2015.

[43] Q. Ai, B. Ding, Q. Liu, and W. Meng, "A subject-specific EMG-driven musculoskeletal model for applications in lowerlimb rehabilitation robotics," International Journal of $\mathrm{Hu}$ manoid Robotics, vol. 13, no. 3, Article ID 1650005, 2016.

[44] J. Han, Q. Ding, A. Xiong, and X. Zhao, "A state-space EMG model for the estimation of continuous joint motions," IEEE Transactions on Industrial Electronics, vol. 62, no. 7, pp. 4267-4275, 2015.

[45] S.-H. Kim, J.-H. Ryu, B.-H. Lee, and D.-H. Kim, "Human identification using EMG signal based artificial neural network," Journal of the Institute of Electronics and Information Engineers, vol. 53, no. 4, pp. 142-148, 2016.

[46] M. G. Jahromi, H. Parsaei, A. Zamani, and M. Dehbozorgi, "Comparative analysis of wavelet-based feature extraction for intramuscular EMG signal decomposition," Journal of Biomedical Physics \& Engineering, vol. 7, no. 4, p. 365, 2017.

[47] Q. Li, J. Peng, D. Tang, and X. Sun, "A study on the UKFNNbased online detection of effluent COD in water sewage treatment," in Proceedings of the 2015 IEEE 10th Conference on Industrial Electronics and Applications (ICIEA), pp. 7-10, IEEE, Auckland, New Zealand, June 2015.

[48] W. Zhou, X. Liang, T. Li, L. Yao, and X. Gu, "Modeling method based on iterative UKFNN pumping oil production process," in Proceedings of the 2017 29th Chinese Control And Decision Conference (CCDC), pp. 4334-4339, IEEE, Chongqing, China, May 2017.

[49] S. Xin and P. Qin, "Model of aluminum electrolysis based on square root unscented Kalman neural network," Chinese Journal of Scientific Instrument, vol. S1, 2016. 